\title{
VARIACIÓN DEL COMPONENTE ANTROPOMÉTRICO Y PARÁMETROS CARDIOVASCULARES DE JÓVENES UNIVERSITARIOS ENTRE 2013 Y 2016 EN POPAYÁN, COLOMBIA
}

Nancy Janneth Molano Tobar ${ }^{1}$, Dolly Ximena Molano Tobar ${ }^{2}$, Raquel Amalia Vélez Tobar $^{3}$

\section{Resumen}

Objetivo. Identificar la variación en el tiempo de algunos parámetros antropométricos y cardiovasculares relacionados con la salud en jóvenes de una Institución de Educación Superior (IES) en Popayán, Colombia, en el periodo comprendido entre 2013 y 2016. Métodos. Estudio cuantitativo de tipo descriptivo-comparativo de carácter longitudinal, con una muestra intencional de 142 estudiantes universitarios, conformada por 70 hombres y 72 mujeres con un promedio de edad de 19,65 años $\pm 1,66$, a quienes se les determinó el IMC, perímetros de cintura y cadera, test de Ruffier y valoración de la presión arterial. Resultados. Hay cambios de 2013 a 2016 en los universitarios. Se relacionan con el incremento de sobrepeso y obesidad, presencia de prehipertensión arterial y baja resistencia cardiovascular ante el ejercicio, con correlaciones significativas para el sexo femenino. Conclusiones. La vida universitaria genera detrimentos en la salud cardiovascular como, por ejemplo, incremento de sobrepeso, lo que demerita seguimiento para prevenir enfermedades futuras.

${ }^{1}$ Fisioterapeuta, Especialista en Docencia Universitaria, Magíster en Educación, Candidata a Doctorado en Ciencias Biomédicas. Docente titular, Universidad del Cauca, Calle 5 № 4-70 Popayán-Cauca-Colombia.najamoto@unicauca.edu.co

2 Economista, Especialista en Finanzas, Magíster en Administración, Docente Universidad Santiago de Cali.xmolanot@gmail.com

${ }^{3}$ Fisioterapeuta, Magíster en Actividad Física. Docente Ocasional Universidad del Cauca.raquelveleztobar@gmail.com 


\section{VARIATION OF THE ANTHROPOMETRIC COMPONENT AND CARDIOVASCULAR PARAMETERS OF YOUNG UNIVERSITY STUDENTS BETWEEN 2013 AND 2016 IN POPAYÁN, COLOMBIA}

Nancy Janneth Molano Tobar ${ }^{1}$, Dolly Ximena Molano Tobar² ${ }^{2}$ Raquel Amalia Vélez Tobar $^{3}$

\section{Abstract}

Objective. To identify the time variation of some anthropometric and cardiovascular parameters related to health in youths of a Higher Education Institution (IES in Spanish) in Popayán-Colombia between 2013 and 2016. Methods. Quantitative study of descriptive and comparative type of longitudinal character, with an intentional sample of 142 university students, consisting of 70 men and 72 women with an average age of 19.65 years \pm 1.66 , who were determined the IMC, waist and hip perimeters, Ruffier Dickson test and of blood pressure valuation. Results. University students have experienced changes between 2013 to 2016, related to overweight and obesity increase, the presence of pre-hypertension and low cardiovascular resistance when working out, with significant correlations for females. Conclusions. University life generates detriments in cardiovascular health as an increase in overweight, which detracts from monitoring in order to prevent future diseases. 


\section{VARIAÇÃO DO COMPONENTE ANTROPOMÉTRICO E PARÂMETROS CARDIOVASCULARES DE ESTUDANTES UNIVERSITÁRIOS ENTRE 2013 E 2016 EM POPAYÁN, COLÔMBIA}

Nancy Janneth Molano Tobar ${ }^{1}$, Dolly Ximena Molano Tobar ${ }^{2}$, Raquel Amalia Vélez Tobar $^{3}$

\section{Resumo}

Objetivo. Identificar a variação no tempo de alguns parâmetros antropométricos e cardiovasculares relacionados à saúde em jovens de uma Instituição de Ensino Superior (IES) em Popayán, Colômbia, no período de 2013 a 2016. Métodos. Estudo quantitativo de tipo descritivo comparativo de carácter longitudinal, com uma amostra intencional de 142 estudantes universitários, composta por 70 homens e 72 mulheres, com uma idade média de 19,65 anos $\pm 1,66$. Determinou-se para eles o IMC, perímetros de cintura e quadril, teste de Ruffier Dickson e avaliação da pressão arterial. Resultados. Há mudanças entre 2013 e 2016 para os universitários, relacionadas ao aumento do sobrepeso e obesidade, presença de pré-hipertensão e baixa resistência cardiovascular ao exercício, com correlações significativas para o sexo feminino. Conclusões. A vida universitária gera prejuízos na saúde cardiovascular como aumento no excesso de peso, o que justifica o monitoramento para prevenir futuras doenças.. 


\section{Introducción}

Los adolescentes universitarios representan un grupo poblacional de relevancia desde diferentes perspectivas, especialmente desde la visión de salud, pues son ellos los futuros adultos y, si se detecta una alteración en el proceso de salud-enfermedad en esta etapa, se generaran posibles repercusiones en la adultez. Es así como Navarro-Prado et al.(1), indica que los universitarios son un "colectivo social en riesgo desde el punto de vista nutricional", pues son ellos los responsables del control de consumo de sus propios alimentos. En muchos casos, salen de sus casas a otras ciudades y se enfrentan a un riesgo, ya que la preferencia es hacia "la dieta rápida, régimen de comidas irregulares, omisión del desayuno, consumo excesivo de alimentos hipercalóricos como de alcohol y otras sustancias tóxicas".

Por lo tanto, se puede establecer que los cambios alimentarios son propicios para el desarrollo de enfermedades crónicas como obesidad, diabetes, hipertensión arterial y enfermedad cardiovascular(2). Por ende, el riesgo para la salud es latente, lo que demuestra que una supervisión de esta situación amerita ser estudiada. A la vez, es relevante identificar el patrón de sobrepeso u obesidad que están presentando los jóvenes universitarios, como su transformación en el trascurso del tiempo de estudio durante sus carreras profesionales, especialmente en las licenciaturas. Por ello, este estudio abarca la importancia de evidenciar como es la evolución de algunos parámetros antropométricos y de salud en jóvenes universitarios entre el periodo 2013 y 2016.
Ledo-Varela et al(3) exponen que "El grupo de trabajo internacional en obesidad (IOTF) y la Organización Mundial de la Salud (OMS) definieron a la obesidad como la epidemia del s. XXI, por su alta prevalencia, evolución ascendente, alto impacto sobre las enfermedades crónicas y aumento del gasto sanitario". Por otro lado, Padilla-García et al(4) ponen de manifiesto que el sobrepeso y la obesidad son factores de riesgo en la etapa universitaria, pues la rutina académica propicia implicaciones positivas $y$ negativas que inciden en las conductas sedentarias que se asocian al aumento de consumo de alimentos hipercalóricos o alimentos industrializados (5), disminución de la práctica de actividad físi$\mathrm{ca}(6)$. Al respecto, Díaz et al(7) reportan la inactividad física en $88 \%$ de los estudiantes de medicina de la Universidad de Valparaíso (Chile). Asimismo, para Colombia, Rangel et al(8) informan que el 77\% de la población de jóvenes realizan "poco o ningún tipo de ejercicio físico durante al menos 30 minutos, con frecuencia de tres veces por semana". Es así como la inactividad física también está relacionada con el uso de la tecnología y con poco tiempo libre para hacer ejercicio debido a sus obligaciones académicas.

La investigación de Chiang-Salgado et al(6), confirma que los estudiantes universitarios presentan cambios en su estilo de vida al ingresar a la universidad y demuestra que tienen un aumento del índice de masa corporal por año de estudio, lo que repercute también en la presencia de prehipertensión arterial. Esta patología es de relevancia para la salud, pues se asocia directamente con 
el síndrome metabólico y con enfermedades cardiovasculares(9); en la población adulta es común su tamizaje pero en niñez y adolescencia la vigilancia que se realiza al respecto es reducida, por lo que esta situación es motivo de alerta, así lo manifiestan Uribe et al(10), al inferir que la pesquisa y prevención de la hipertensión arterial producen una disminución en la prevalencia de enfermedades cardiovasculares, en especial en la población infantil y joven, confiriendo de esta manera una posibilidad de disminuir a futuro el índice de muertes a causa de estos factores. De la misma manera, Bautista et al(11), presentan una positiva relación entre hipertensión arterial y obesidad, lo que demuestra la necesidad de estudiarlo.

Otro parámetro complementario y un indicador notable de salud de una población relacionada con el sobrepeso y la obesidad es el índice de cintura-cadera que constituye el indicador antropométrico más preciso a considerar en la valoración de la grasa corporal total y la masa grasa intra-abdominal(12). Según Magalhães et al(13), este indicador facilita la identificación del riesgo cardiovascular por la ubicación de la adiposidad central. Adicionalmente, Pérez et al(14), manifiestan una relación positiva entre el sedentarismo y la aparición del sobrepeso y la obesidad en población de 2 a 24 años.

La falta de identificación y seguimiento de los procesos de salud en los jóvenes conlleva la presencia de enfermedades en el adulto. Por este motivo, este trabajo tuvo como objetivo principal identificar la evolución que presentaban algunos parámetros antropométricos y de salud en los jóvenes universitarios de la ciudad de Popayán, Colombia, en el periodo comprendido entre 2013 y 2016. A su vez, pretendemos realizar una identificación de factores de riesgo que permitieran determinar a futuro pautas de trabajo en pro de la salud de la población.

\section{Material y métodos}

El estudio tuvo un enfoque cuantitativo, de tipo descriptivo-comparativo con diseño longitudinal, debido a que se realizó entre los años 2013 y 2016, donde utilizó una muestra intencional representada en 142 estudiantes de la Licenciatura en Educación Física, Recreación y Deportes de una institución universitaria de educación superior del suroccidente del país, distribuidos en 70 hombres y 72 mujeres con un promedio de edad de 19,65 $\pm 1,66$. Los criterios de inclusión correspondieron a que los participantes presentaran el consentimiento firmado por ellos si eran mayores de edad o por sus tutores, en el caso de los menores de edad. De la misma manera, se admitían aquellos participantes que presentaran registros completos de la primera evaluación (2013), aquellos que estuvieran matriculados académicamente y que no presentaran patologías psiquiátricas que afectaran el desarrollo de la segunda evaluación (2016). La recolección de los datos se realizó en dos momentos: el primero, entre enero al mes de diciembre de 2013, donde se incluyeron datos de estudiantes de primero y quinto semestre; y el segundo momento, entre febrero de 2016 al mes de noviembre de 


\section{Procedimiento}

La evaluación estuvo a cargo del personal médico que apoyó el proyecto. Las variables que se evaluaron tuvieron en cuenta los estándares de la Sociedad Internacional para el Avance de la Kineantropometría presentados por Pérez et al(14), que consisten en la medición de la talla en un estadiómetro, con escala métrica de $1 \mathrm{~mm}$ de precisión, y para el peso corporal total, se emplea una balanza marca Detecto con precisión de 100gr. Para el cálculo del índice de masa corporal (IMC), se tuvo en cuenta las sugerencias de Valverde et al(15), quien calcula el IMC a partir de la fórmula peso/altura2. La escala de evaluación que se tuvo en cuenta es la especificada por la OMS (16), donde expresan las categorías así: "IMC se realiza con el siguiente sistema de categorías: Insuficiente (IMC $<18.5)$, Normal $(\geq 18.5 \mathrm{y}<25)$, Sobrepeso $(\geq 25 \mathrm{y}$ $<30)$ y Obesidad $(\geq 30)$ ".

Para la medición de la presión arterial se utilizó un tensiómetro digital marca Omron. La presión arterial fue medida en el brazo derecho y estando sentado. Se tomó en 3 ocasiones, con intervalos de 20 minutos para que el sujeto descansara. Los resultados se ubicaron en tablas de clasificación de la presión arterial (PA)(17). Los rangos tenidos en cuenta corresponden a la presión sistólica con valores $\geq 140 \mathrm{mmHg}$ y la presión diastólica con valor de $\geq 90 \mathrm{mmHg}(18)$. La clasificación se define según la Guía colombiana para el diagnóstico y tratamiento de la hipertensión arterial (19).

Para determinar el riesgo cardiovascular se realizó el test de Ruffier(20), que per- mite determinar la adaptación cardiovascular al esfuerzo. La prueba consiste en medir la frecuencia cardiaca en diferentes momentos "La primera medición es después de 5 minutos de descanso y es registrado como PI, luego los estudiantes hacen 30 sentadillas por $45 \mathrm{~s}, \mathrm{y}$ el pulso se mide nuevamente por $15 \mathrm{~s}$, este resultado se registra como P2. Después de eso, se da una recuperación de 1 minuto y el pulso, medido de nuevo, se registra como P3"(21). Los resultados obtenidos se aplican a la formula índice de Dickson $=((\mathrm{P} 2-70)+2(\mathrm{P} 3-\mathrm{P} 1)) / 10$, indicando excelente adaptación (menor a 0 ), muy buena adaptación (1-5), buena adaptación (5,1-10), adaptación insatisfactoria $(10,1-15)$ y mala adaptación (15,1 o más).

La circunferencia de cintura $(\mathrm{cm})$ se mide según los criterios del National Cholesterol Education Program Adult Treatment Panel III (NCEP ATP III), donde, ante el riesgo para desarrollar complicaciones metabólicas relacionadas con la obesidad, se clasificaron los valores $>102 \mathrm{~cm}$ en hombres $\mathrm{y}>88 \mathrm{~cm}$ en mujeres como riesgo sustancialmente aumentado, los valores $\geq 94$ y $<101,9$ en hombres y $\geq 80$ y $<88$ como riesgo aumentando, los valores $<94$ en hombres $\mathrm{y}<80$ en mujeres se clasificaron como riesgo bajo"(22).

Asimismo, y de acuerdo con González et al.(12), "fueron evaluados los perímetros de la cintura y la cadera, para lo que se utilizó una cinta métrica flexible e inextensible, cuya precisión es de $1 \mathrm{~mm}$ ". La circunferencia de cintura se tomó entre la última costilla y la cresta ilíaca, con el sujeto de pie y respiración 
normal. La circunferencia de la cadera se tomó midiendo a nivel de los trocánteres mayores, coincidiendo con la sínfisis pubiana; para ello el sujeto debía estar de pie, con los glúteos relajados y los pies juntos". De la evaluación de los perímetros de cintura y cadera se establece, según Campos-Mondragón(23), "se identificó a los sujetos con riesgo de síndrome metabólico: mujeres: $\geq 0,85$; hombres: $\geq 0,90$ ".

Las evaluaciones se sistematizaron en una base de datos con el programa SPSS versión 20.0 para Windows. Se procedió a calcular medidas de tendencia central (media), medidas de dispersión absoluta (desviación de medias) y medidas de dispersión relativas (comparación de medias ANOVA y la utilización de estadísticos para identificar los niveles de correlación), considerándose diferencias estadísticamente significativas los valores de $\mathrm{P}<0.05$.

\section{Aspectos ético-legales}

Se tuvo en cuenta lo dispuesto en la Declaración de Helsinki(24) y los dispuesto en la Resolución 8430 del Ministerio de Salud y Protección Social de Colombia(25) para investigación con personas. Al grupo de apoyo para la investigación se le ofrecieron charlas sobre los aspectos éticos y los procedimientos para los abordajes y encuentros. Tras esto, se presentó el proyecto a los padres de familia y estudiantes, previo aval del comité de ética (R-035-2002) de la Universidad del Cauca. A la población y tutores se les garantizó la protección a la intimidad, a la confidencialidad y al derecho al anonimato de los datos suministrados en las evaluaciones, según lo dispuesto en la Ley 1581 de 2012, considerando el estudio con riesgo mínimo de lesión para los participantes.

\section{Resultados}

Las características sociodemográficas de los estudiantes demostraron que la mayoría provienen del estrato cuatro $(30,3 \%)$ de la ciudad de Popayán, y su edad promedio es de 19,65 años $\pm 1,66$; se han mantenido en la carrera por alrededor de 5 años en total. Con relación a los parámetros de salud para la primera evaluación de 2013, se evidenció que la población presentó una presión arterial sistólica de 104,96 mmhg $\pm 11,67$, y una presión diastólica de 62,98 mmhg $\pm 9,53$, se consideró que estaban dentro de los valores normales. Para los resultados de 2016 se evidenciaron cambios de presión arterial sistólica de 112,96 hmmg $\pm 11,67$ y presión diastólica de $72,98 \mathrm{hmmg} \pm 9,53$; se reveló un aumento comparativo para los dos periodos.

Según los resultados obtenidos del test de Ruffier- en el 2013, una evaluación de $7,33 \pm 3,38$ indicó de acuerdo con la escala de valoración que los estudiantes de la licenciatura tienen en general una buena adaptación. No obstante, dicho parámetro cambió para el 2016, dado que se

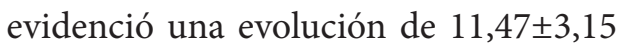
que mostró una adaptación insatisfactoria. El gráfico ayudó a diferenciar los resultados por sexo; las mujeres se encuentran con una adaptación al ejercicio inferior a la de los hombres. 


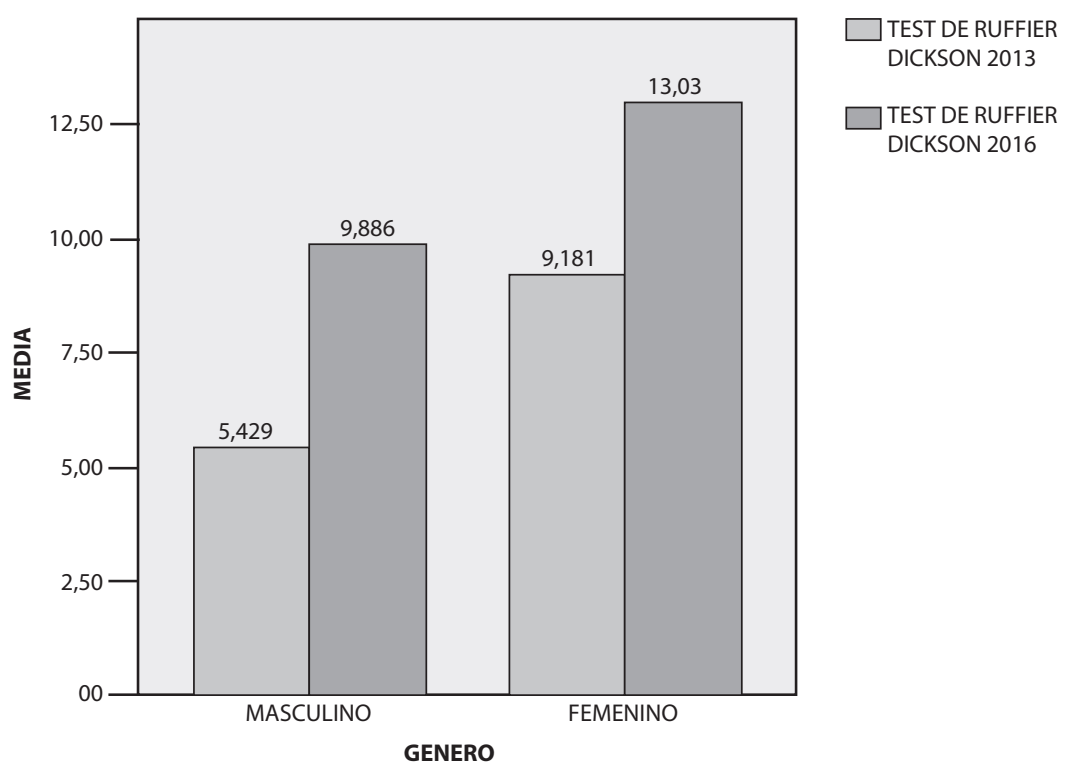

Figura 1. Diferencia por sexo en el test de Ruffier

Las mediciones antropométricas se muestran en la Tabla 1. El índice de masa corporal estaba en normalidad $(22,4 \mathrm{~kg} /$ $\mathrm{mt} 2 \pm 3,0)$ en el 2013, y en el 2016 la situación cambió, puesto que se generó un aumento y, teniendo en cuenta la clasificación de la OMS, se clasificó el nivel como uno de sobrepeso, 25,1 $\pm 2,2$. La situación se refleja en forma similar tanto para hombres como para mujeres.

Tabla 1. Evolución de los parámetros antropométricos 2013-2016

\begin{tabular}{|c|c|c|c|c|c|c|c|c|c|}
\hline \multicolumn{2}{|c|}{ SEXO } & $\begin{array}{l}\text { IMC } \\
2013\end{array}$ & $\begin{array}{l}\text { IMC } \\
2016\end{array}$ & $\begin{array}{c}\text { Perímetro } \\
\text { de Cintura } \\
2013\end{array}$ & $\begin{array}{c}\text { Perímetro } \\
\text { de Cintura } \\
2016\end{array}$ & $\begin{array}{c}\text { Perimetro } \\
\text { Cadera } \\
2013\end{array}$ & $\begin{array}{c}\text { Perímetro } \\
\text { Cadera } \\
2016\end{array}$ & $\begin{array}{c}\text { ICC } \\
2013\end{array}$ & $\begin{array}{c}\text { ICC } \\
2016\end{array}$ \\
\hline \multirow[b]{2}{*}{ Masculino } & Media & 22,4 & 25,2 & 73,9 & 81,5 & 92,7 & 97,3 & 0,80 & 0,84 \\
\hline & $\begin{array}{l}\text { Desviación } \\
\text { estándar }\end{array}$ & 2,9 & 2,6 & 9,2 & 7,8 & 3,6 & 6,3 & 0,07 & 0,05 \\
\hline \multirow[b]{2}{*}{ Femenino } & Media & 22,3 & 25,0 & 66,5 & 72,6 & 92,9 & 101,6 & 0,72 & 0,72 \\
\hline & $\begin{array}{l}\text { Desviación } \\
\text { estándar }\end{array}$ & 3,1 & 1,8 & 6,8 & 6,0 & 5,6 & 5,4 & 0,06 & 0,06 \\
\hline \multirow[b]{2}{*}{ Total } & Media & 22,4 & 25,1 & 70,1 & 77,0 & 92,8 & 99,5 & 0,75 & 0,78 \\
\hline & $\begin{array}{l}\text { Desviación } \\
\text { estándar }\end{array}$ & 3,0 & 2,2 & 8,8 & 8,3 & 4,7 & 6,2 & 0,08 & 0,08 \\
\hline
\end{tabular}


El perímetro de cintura en el 2013 se encontró en $70,1 \mathrm{~cm} \pm 8,8$; en 2016 aumentó hasta un $77,0 \pm 8$, y se mantuvo dentro de los parámetros de normalidad. Respecto al sexo, quienes presentaron mayor perímetro de cintura en 2013 fueron los hombres, con $73,9 \mathrm{~cm} \pm 9,2$ mientras que las mujeres presentaron $66,5 \mathrm{~cm} \pm 6,8$. En el 2016, se evidencia un aumento para los dos sexos: el promedio de los hombres estuvo en $81,5 \mathrm{~cm} \pm 7,8$, que es considerado como de bajo riesgo; en las mujeres se registró un $72,6 \mathrm{~cm} \pm 6,0$.
El índice de cintura cadera en la población estuvo bajo los parámetros de normalidad tanto para 2013 como para $2016(0,75 \pm 0,08$ vs $0,78 \pm 0,08)$ y, según el sexo, se observó que los hombres tuvieron una relación superior $(0,80 \pm 0,007)$ a la de las mujeres $(0,72 \pm 0,06)$. Para las variables evaluadas se quiso establecer la relación entre las mismas variables y cruzarlas de acuerdo con el sexo con un $\mathrm{P}<0,05$, estableciendo que se plantea en la Tabla 2.

Tabla 2. Nivel de significancia entre variables y sexo

\begin{tabular}{lcccc}
\hline \multicolumn{1}{c|}{ Variables } & Total & Masculino & Femenino \\
\hline IMC 2013/IMC 2016 & 0,000 & 0,000 & 0,021 \\
Perímetro cintura 2013/ Perímetro de cintura 2016 & 0,000 & 0,000 & 0,000 \\
Perímetro de cintura 2013/ Presión arterial 2016 & 0,000 & 0,000 & 0,000 \\
Perímetro cintura 2013/Perímetro cadera & 0,000 & 0,000 & 0,000 \\
ICC 2013/ ICC 2016 & 0,000 & 0,000 & 0,072 \\
Test Ruffier 2013/ Test Ruffier 2016 & 0,000 & 0,493 & 0,000 \\
Presión arterial 2013/ Presión arterial 2016 & 0,000 & 0,000 & 0,000
\end{tabular}

Al analizar las variables de salud de acuerdo con la evolución que los estudiantes manifestaron, en relación con la presión arterial, se encontró que el número de estudiantes con presión arterial normal era 131 y en la evaluación del 2016 el número era 128. Por ende, se incrementaron los diagnósticos para los parámetros de prehipertensión ( $\mathrm{n}=8$ vs $\mathrm{n}=11$ ), e hipertensión arterial estadio II, pasando de 0 estudiantes a 1 . Tales manifestaciones se observaron en los últimos semestres de la carrera.
Una situación similar se presentó al evaluar el test de Ruffier- en los semestres, notando que, si bien 43 estudiantes tenían muy buena adaptación en 2013, en la evaluación del 2016 dicha calificación solamente se presentó en un estudiante. Para el resto de calificaciones aumentaron en deficiencia para el 2016, mostrando que el ítem de adaptación insatisfactoria pasó de 23 a 74 y el de adaptación mala, que estaba en cero en el 2013, pasó a manifestarse en 13 personas en el 2016. De este modo, en quienes se 
presentaron mayores cambios fueron en los estudiantes de primeros semestres.

El panorama planteado para los aspectos antropométricos no varía en las condiciones anteriormente planteadas, ya que para el IMC se observó que los estudiantes que se encontraban en normalidad en el 2013 (112) pasaron a ser solo 60 en el 2016. Entonces, se distribuyeron en los indicadores de sobrepeso en el 2013 y 2016, respectivamente, (25 vs 77). Al revisar el nivel de significancia de las variables con las variables por semestre, ninguno presentó significancia estadística.

\section{Discusión}

$\mathrm{Al}$ analizar la evolución de las variables propuestas, se logra evidenciar que los jóvenes se identifican sociodemográficamente con otros estudios similares en diferentes contextos universitarios de índole pública y tienden a cambiar a lo largo de la vida universitaria. Dichos cambios se asocian a los rangos de la población en general por su promedio de edad, como lo plantea Ramírez-Vélez et al(26), al situar a los jóvenes universitarios en una edad promedio de 20,3 años. Por su edad, tienden a tomar acciones apresuradas respecto a su estilo de vida. Por el contrario, Padilla-García et al(4) infieren que los cambios se presentan debido a que los jóvenes no tienen la destreza para afrontar lo que la vida les presenta, así que se generan incrementos or factores de riesgo que afectan la salud. Ello se puede asociar con los cambios en los estilos de vida de las poblaciones(27). De la misma manera, Sampaio et al(28), evidencia como los jóvenes que viven con sus padres presentan menos riesgo en cuanto a la nutrición y desarrollo de inadecuadas prácticas relacionadas con la salud-enfermedad, hecho que se pudo visualizar en este trabajo.

En cuanto a las condiciones de salud de los jóvenes universitarios, se apreció que la población presentó normalidad en las variables de presión arterial para 2013, mientras que para el 2016, se apreció una modificación, resaltando estudiantes con prehipertensión, lo que supone un riesgo a medida que avanzan en la edad y en su carrera. Este hecho debe ser asociado a factores como la calidad nutricional o componentes genéticos que pueden inferir en esta manifestación. Así lo sustenta Uribe- et al(10), y añaden que es necesario implementar estrategias de prevención y seguimiento a modificaciones en la presión arterial en niños y adolescentes para prevenir problemas de salud a futuro. Esto se complementa con lo planteado por Gonzales-Jiménez et al(12) quienes determinan que la presión arterial son un indicativo de salud pública que incide a futuro en el aumento de enfermedades cardiovasculares, lo que se asocia con las medidas antropométricas encontradas en la población.

Respecto a la presión arterial según el sexo, se evidenció que la población femenina fue la que presentó mayores incrementos respecto a la población masculina, situación opuesta a la encontrada por Bautista et al(11), explicado desde el aumento del endurecimiento de las arterias de gran tamaño. Sampaio et al(28), demostraron que la hipertensión arterial empieza a los 17,8 años, edad que se 
asocia con la población de este estudio, lo que indica la necesidad de continuar realizando seguimientos de índole preventivo -que ahora se realizan con menor frecuencia- a nuestros estudiantes universitarios, más sabiendo que son estudiantes de licenciatura en educación física, recreación y deporte. La evidencia demostró una asociación directa entre la hipertensión arterial con el incremento del perímetro de cintura, lo cual justifica que los estudiantes están en mayor riesgo en salud, como lo sustenta Múnera et al(29) al determinar que los inadecuados hábitos de vida representan una fuente de riesgo en salud.

Los datos del test de Ruffier lograron determinar que la población presentó un aumento en los dos periodos evaluados, lo que evidencia un detrimento en la resistencia del sistema cardiaco ante el ejercicio. Este hecho permite considerar que, a medida que avanza la edad, dicha condición desmejora. Por otra parte, se evidenció que las mujeres presentaron bajos niveles de resistencia cardiovascular tanto en el primer como en el segundo periodo. Este fue un escenario similar al presentado por Peltekova(21) y confirmado por Rodríguez et al(20). Se infiere que los chicos realizan mayor ejercicio que las chicas, lo que repercute positivamente en la resistencia cardiovascular.

Para el IMC, se encontró que la población universitaria se encontraba con indicadores de normalidad en las dos valoraciones. No obstante, los valores del IMC aumentaron con relación al tiempo, es decir, a medida que avanza la edad, se incrementa el peso corporal que puede conllevar a un sobrepeso y obesidad, lo que se correlaciona con los datos informados por Oleas et al(30). Igualmente, se logró demostrar que las mujeres, en las dos mediciones, están por encima de los hombres, lo que se corrobora en otra investigación(31), donde se infiere que las mujeres tienen tendencias mayores hacia el sedentarismo, que se correlaciona con altos niveles de sobrepeso y obesidad, caso opuesto en una investigación con población chilena (31) donde los hombres manifestaron mayores valores de IMC, debido a cambios en los niveles de estilo de vida de la población con tendencia al sedentarismo.

Por otro lado, se observó que el perímetro de cintura incrementó en la población de la primera evaluación a la segunda, al igual que el ICC. De este modo, fue posible asociar estos resultados con otros estudios (5), donde se permite evidenciar que la grasa abdominal es un significado de riesgo cardiovascular, como el desarrollo y asociación positiva al síndrome metabólico, altamente manifestado en diversas investigaciones(32) relacionadas con la salud pública de un país y el latente riesgo en salud de una población como la explorada en este trabajo.

Se demostró que existe un nivel de significancia en ciertas variables de índole antropométrico. Esto representa una evidencia como advertencia a la población universitaria quienes deben velar más por realizar actividad física durante sus procesos académicos, pues sus índices de correlación son semejantes a los encontrados en otros estudios (33) que demuestran el riesgo de salud y la necesidad de generar estrategias para prevenir enfermedades derivadas del sedentaris- 
mo y de la poca actividad física, en especial para la población femenina.

\section{Conclusiones}

Existen cambios importantes relacionados con los parámetros antropométricos en los universitarios, desde el inicio de su carrera hasta el desarrollo de la misma, lo cual genera un precedente para propiciar estrategias de prevención y de promoción de la salud en esta población. Hay evidencia del incremento de prehipertensión arterial y de una mala resistencia cardiovascular al ejercicio en

\section{Referencias}

1. Navarro-Prado S, González-Jimenez $\mathrm{E}$, Montero-Alonso $\mathrm{M}$, et al. Estilo de vida y seguimiento de la ingesta dietética en estudiantes del Campus de la Universidad de Granada en Melilla. Nutr Hosp. 2015;31(6):2651-9.

2. Bencomo $M$, Dugarte $N$, Berríos $A$, Blanco M. Factores de riesgo de obesidad y sobrepeso en el personal docente universitario. Barquisimeto Estado Lara. Venezuela. Salud, arte y cuidado. 2011;4(2):20-31.

3. Ledo-Varela M, de Luis Román D, González-Sagrado $M$, et al. Características nutricionales y estilo de vida en universitarios. Nutr Hosp. 2011;26(4):814-8.

4. Padilla-García $\mathrm{Cl}$, Jaimes-Valencia ML, Fajardo-Nates S, Ramos-Franco AM. Factores de riesgo cardiovascular y estilos de vida de estudiantes universitarios. Med UNAB [Internet]. 2014;17(2):81-90. Disponible en:

$20 \mathrm{http}: / /$ search.ebscohost.com/login.aspx?direct=true \&db=fua\&AN=111794807\&lan$g=e s \&$ site=ehost-live los jóvenes universitarios, con mayor presencia en la población femenina. Por ende, es importante desarrollar acciones que involucren mayor actividad física y controles hacia hábitos de vida saludable, con el fin de reducir las posibles tasas de enfermedades cardiovasculares.

Impulsar acciones de salud en estudiantes de Licenciatura en Educación Física, Recreación y Deporte demerita no solo un conocimiento sino una puesta en marcha de comportamientos saludables que permitan mejorar y proteger su propia salud y la de los demás.

5. Molano-Tobar NJ, Ordoñez-Fernández MY, Molano-Tobar DX. Cambios antropométricos y asociación del nivel de actividad física en docentes universitarios. Revista Ciencia y Cuidado. 2017;14(2):39-51.

6. Chiang-salgado MT, Casanueva-escobar V, González-Rubilar U, et al. Factores de riesgo cardiovascular en estudiantes universitarios chilenos. Salud Pública Mex. 1999;41:444-51.

7. Díaz S, González F, Arrieta K. Niveles de actividad física asociados a factores sociodemograficos, antropométri$\cos$ y conductuales en universitarios de Cartagena (Colombia). Salud Uninorte. 2014;30(3):405-17.

8. Rangel L, Rojas L, Gamboa E. Sobrepeso y obesidad en estudiantes universitarios colombianos y su asociación con la actividad física. Nutr Hosp. 2015;31(2):629-36.

9. González C, Díaz Y, Mendizabal-Ruiz A, et al. Prevalencia de obesidad y perfil 
lipídico alterado en jóvenes universitarios. Nutr Hosp. 2014;29(2):315-21.

10. Uribe L, Spencer L, Fritsch A, et al. Prevalencia de hipertensión arterial en alumnos de $4^{\circ}$ básico a $1^{\circ}$ medio en un colegio de Santiago (Chile). Salud Uninorte. 2013;29(2):214-25.

11. Bautista L, Vera-Cala LM, Villamil L, et al. Factores de riesgo asociados con la prevalencia de hipertensión arterial en adultos de Bucaramanga, Colombia. Salud Pública Mex. 2012;44(29):399-405.

12. González-Jiménez E, Montero-Alonso M, Schmidt-Riovalle J. Estudio de la utilidad del índice de cintura-cadera como predictor del riesgo de hipertensión arterial en niños y adolescentes. Nutr Hosp. 2013;28(6):1993-8.

13. Magalhães E, Santana L, Priore $S$, Franceschini S. Perímetro da cintura, relação cintura/estatura e perímetro do pescoço como parâmetros na avaliação da obesidade central em crianças. Rev Paul Pediatr. 2014;32(3):273-82.

14. Pérez $B$, Landaeta-Jiménez $M$, Arroyo $E$, Marrodán MD. Patrón de actividad física, composición corporal y distribución de la adiposidad en adolescentes venezolanos. An Venez Nutr. 2012;25(1):5-15.

15. Valverde P, Rivera F, Moreno C. Diferencias de sexo en imagen corporal, control de peso e índice de masa corporal de los adolescentes españoles. Psicothema. 2010;22:77-83.

16. Salazar CM, Feu S, Vizuete M, de la Cruz-Sánchez E. IMC y actividad física de los estudiantes de la Universidad de Colima. Rev Int Med y Ciencias la Act Física y el Deport [Internet]. 2013;13(51):569-84. Disponible en:

http://cdeporte.rediris.es/revista/revista51/artIMC405.pdf

17. Chobanian A, Bakris G, Black $H$, et al. Séptimo informe del comité nacional conjunto en prevención, detección, eva- luación y tratamiento de la hipertensión arterial. Hypertension. 2003;42:1206-52.

18. Power C, Pinto S, Law C, Ki M. Obesity and risk factors for cardiovascular disease and type 2 diabetes: Investigating the role of physical activity and sedentary behaviour in mid-life in the 1958 British cohort. Artherosclerosis [Internet]. 2014;233:363-9. Disponible en: https:// www.ncbi.nlm.nih.gov/pubmed/24530764

19. Argemiro Fragozo, MD.; Pablo Aschner MD., MSc.; Juan B. Pinzón, MD., MSc.; Efraín Gómez M. Guías colombianas para el diagnóstico y tratamiento de la hipertensión arterial. Revista Colombiana De Cardiología. 2009. (REVISAR)

20. Rodríguez M, García A, García T, et al. Actividad física y ocio y su relación con el índice de Ruffier en adolescentes. Rev Int Cienci Act fis Deport. 2015;15(57):16580.

21. Peltekova I. Assessment of the level of preparation of students through the Ruffier and Dickson tests. Act Phys Educ Sport. 2017;7(1):35-8.

22. Hall Lopez J, Monrecal L, Ochoa PY, Vega JL. Nivel de actividad física, estado nutricional y obesidad abdominal en docentes de la escuela superior de Educación Física-UAS. Ciencia, Deport y Cult Física. 2007;1(3):12-5.

23. Campos-Mondragón M. Obesidad y riesgo de síndrome metabólico en estudiantes de posgrado de Veracruz, México. Rev Española Nutr Humana y Dietética [Internet]. 2015;19(4):197. Disponible en: http://www.renhyd.org/index.php/renhyd/ article/view/170

24. Declaración de Helsinki. Principios éticos para las investigaciones médicas en seres humanos. Tokio-Japón: Asociación Médica Mundial; 1975 p. 5.

25. Ministerio de Salud y Protección Social Colombia. Resolución 8430. 1993. p. 1-12. 
26. Ramírez-Vélez R, Triana-Reina HR, Carrillo HA, Ramos-Sepúlveda JA. Percepción de barreras para la práctica de la actividad física y obesidad abdominal en universitarios de Colombia. Nutr Hosp. 2016;33(6):1317-23. Disponible en:

http://revista. nutricionhospitalaria.net/index.php/nh/article/view/777

27. Rodríguez F, Espinoza L, Gálvez J, et al. Estado nutricional y estilos de vida en estudiantes universitarios de la Pontificia Universidad Católica de Valparaíso. Universidad y Salud. 2013;15(2):123-35.

28. Sampaio $F$, de Oliveira $P$, da Mata $L$, et al. Profile of nursing diagnoses in people with hypertension and diabetes. Investig y Educ en Enferm. 2017;35(2):139-53.

29. Múnera H, Salazar DA, Pastor MP, Alzate T. Overweight and Obesity Conditions: Prevalence and Associated Risk Factors in Nursing Students in a Public University in Medellín, Colombia. Investig y Educ en Enfermería [Internet]. 2017;35(2):191-8. Disponible en: http://www.redalyc.org/articulo.oa?id=105251300008

30. Oleas M, Barahona A, Salazar R. Índice de masa corporal y porcentaje de grasa en adultos indígenas ecuatorianos Awá. Arch Latinoam Nutr [Internet].
2017;67:42-8. Disponible en: http://www. sciencedirect.com/science/article/pii/ S1886658105761107

31. Vélez C, Vidarte J, Parra J. Niveles de sedentarismo en población entre 18 y 60 años en Manizales, Pereira y Armenia, Colombia. Análisis multivariado. Aquichan [Internet]. 2014;14(3):303-15. Disponible en:

http://aquichan.unisabana.edu.co/index. php/aquichan/article/view/3654/pdf

Vidarte-Claros J a., Vélez-Álvarez C, Parra-Sánchez JH. Niveles de sedentarismo en población de 18 a 60 años. Manizales, Colombia. Rev salud pública [Internet]. 2012;14(3):417-28. Available from: http://www.scielosp.org/pdf/rsap/v14n3/ v14n3a05.pdf

32. Paternina-de la Ossa A, Villaquirán-Hurtado $A$, Jácome-Velasco $S$, et al. Actividad física en pacientes con diabetes mellitus tipo 2 y relación con características sociodemográficas, clínicas y antropométricas. Rev Univ y Salud. 2018;20(1):72-81.

33. Guerrero N, Muñoz R, Muñoz A, et al. Nivel de sedentarismo en los estudiantes de fisioterapia de la Fundación Universitaria María Cano, Popayán. Hacia la Promoción de la Salud. 2015;20(2):78-90. 